\title{
Lineer ve Lineer Olmayan Flor Katkılanmış Lityum Topaklarının Optik Özelliklerinin İncelenmesi
}

\section{The Investigation of Optical Properties of Linear and Non-linear Fluorine-Doped Lithium Clusters}

\author{
Arslan Ünal ${ }^{1 *}$, Selçuk Güvenir ${ }^{2}$ \\ Geliş / Received: 07/07/2019 \\ Revize / Revised: 20/08/2019 \\ Kabul / Accepted: 20/08/2019
}

$\ddot{O}$

z- Bir flor atom katkılanmış lityum topaklarının lineer $\left(\operatorname{Lin}_{n} F, n=1-8\right)$ ve lineer olmayan $\left(\operatorname{Li}_{n} F, n=2-8\right)$ kararlı yapılarının optik özellikleri, hibrit-Yoğunluk Fonksiyonel Teorisi (h-DFT) yardımıyla kuramsal olarak araştırıııışır. Lineer $\mathrm{LinF}(\mathrm{n}=1-8)$ topaklarının en kararlı izomerik yapıları Becke 3 Lee-Yang-Parr (B3LYP) fonksiyoneli ve Los Alamos National Laboratory -2 double zeta (Lanl2dz) temel seti kullanılarak elde edilmiştir. Literatürde rapor edilen lineer olmayan $\operatorname{Lin} F(n=2-8)$ topaklarının taban durumu geometrik yapıları $h$ DFT / B3L YP / Lanl2dz teori seviyesinde kararlıkları test edilmiştir. Elde edilen lineer ve lineer olmayan topakların global minimum yapılarına ait dipol moment $(\mu)$, statik ortalama polarizebilite $(<\alpha>)$, anizotropik polarizebilite $(\Delta \alpha)$ ve birinci dereceden statik toplam moleküler hiperpolarizebilite $\left(\beta_{0}\right)$ değerleri yine ayn metot ve temel set ile incelendi. Bu çalışma, yeni lineer ve lineer olmayan optik malzemelerin veya uygulamaların tasarımında çalşsan deneysel araştırmacılara faydalı optik bilgiler verebilir.

Anahtar Kelimeler- Topak, Lityum, Flor, DFT, Optik Özellikler

bstract- The optical features of linear $\left(\operatorname{Li}_{n} F, n=1-8\right)$ and non-linear $\left(\operatorname{Lin}_{n} F, n=2-8\right)$ stable structures of fluorine-doped lithium clusters were theoretically investigated with the help of hybrid-Density Functional Theory (h-DFT). The most stable isomeric structures of linear $\operatorname{Lin} F(n=1-8)$ clusters were obtained by using the Becke 3 Lee-Yang-Parr (B3LYP) functional and the Los Alamos National Laboratory -2 double zeta (Lanl2dz) basis set. The stabilities of ground state geometric structures of non-linear $\operatorname{Lin}_{n} \mathrm{~F}(n=2-8)$ clusters reported in the literature were tested at $h$-DFT / B3LYP / Lanl2dz level of theory. The dipole moment $(\mu)$, static mean polarizability $(<\alpha>)$, anisotropic polarizability $(\Delta \alpha)$ and molecular first order static total hyperpolarizability $\left(\beta_{0}\right)$ values of the obtained global minimum structures of linear and non-linear clusters were investigated with the same method and basis set. This study may give beneficial optical knowledge to the experimental researchers working in the design of new linear and non-linear optical materials or optical applications.

Keywords- Cluster, Lithium, Fluorine, DFT, Optical Properties

1*Sorumlu yazar iletişim: arslan.unal@bilecik.edu.tr (https://orcid.org/0000-0002-5857-7318)

Fizik Bölümü, Bilecik Şeyh Edebali Üniversitesi, Fen Edebiyat Fakültesi, 11210, Bilecik

2İletişim: selcuk.guvenir@bilecik.edu.tr (https://orcid.org/0000-0001-6322-988X)

Fizik Anabilim Dalı, Bilecik Şeyh Edebali Üniversitesi, Fen Bilimleri Enstitüsü, 11210, Bilecik 


\section{I.GİRIŞ}

Lineer olmayan bir halojen atom katkılanmış küçük lityum topakları $\mathrm{Li}_{\mathrm{n}} \mathrm{X}$ ( $\mathrm{n}>1$ ve $\mathrm{X}=\mathrm{Cl}, \mathrm{Br}, \mathrm{F}$ veya $\mathrm{I}$ ) son on yılda deneysel ve kuramsal topak çalışan bilim adamları tarafından birçok endüstri ve sanayi uygulamalarında örneğin lityum bazlı pil, soğutma, optik sensör, nanotüp, elektrokatalizör, elektrid, kurutucu, iletişim ve veri depolama sistemlerinde süper tuz olarak potansiyelini araştırmaktadır [1-10]. Bu topaklar alkali metal atomu olan lityumdan daha düşük iyonlaşma enerjisine sahip olduğu için süperalkali topak olarak adlandırılmıştır [5, 8, 12-16]. Süperalkali topaklarda, saf metal topaklarına bir halojen atomu eklendiğinde oluşan yeni topakların moleküler yapı geometrilerinde, kararlılıklarında, elektronik özelliklerinde, rölatif enerjilerinde, bağlanma doğaları ve kutuplanabilirlikleri temel değişiklikler meydana gelmektedir.Termodinamiksel olarak kararlı olan bu yeni topaklar hipervalent topaklar olarak da adlandırılır ve $\mathrm{X}_{\mathrm{n}+1} \mathrm{Y}$ deneysel formülündedirler. Burada $\mathrm{X}$ elektronegatif atom ve $\mathrm{Y}$ ise bir elektropozitif veya metal atomudur [14].

Hipervalent ve süperalkali özelliğine sahip bir halojen atom katkılanmış lityum topaklarının termodinamik kararlı olmasının sebebi, lityum kafesi içindeki lityum atomlarının birbirleriyle kovalent bağlanmaları ile anyonik halojen atomların katyonik lityum topak kafesiyle elektrostatik etkileşme içerisinde olmasından dolayıdır [4]. Bu bağlanma doğasına sahip süperalkali tuzlar çok önemli lineer ve lineer olmayan optik özelliklere sahip olabilirler. Literatürde günümüze kadar tek flor atom katkılanmış lityum topakları üzerine çok az sistematik çalışmalar mevcuttur. Moreira ve ark., flor-lityum topaklarını kuantum Monte Carlo yöntemini kullanarak enerjisel özellikleri üzerine bir çalışma yapmışlardır [11]. Lanaro ve Patey lityum halojenürlerin yığın faz ve topak olarak kristal yapıların oluşumlarını kuramsal olarak incelemişlerdir [10]. Dustebek ve ark., $\mathrm{Li}_{n} \mathrm{~F}$ (n=2-6) topaklarının iyonlaşma enerjilerini Knudsen-hücre kütle spektrometresiyle deneysel olarak elde etmişlerdir [9]. Velickovic ve ark., kuramsal ve deneysel olarak $\mathrm{Li}_{\mathrm{n}} \mathrm{F}(\mathrm{n}=2-4)$ topaklarının iyonlaşma enerjilerini araştırmasını gerçekleştirmişlerdir [7]. Ünal ve Kotan çalışmalarında $\mathrm{Li}_{n} \mathrm{~F}(\mathrm{n}=1-8)$ topakların kararlı yapılarını ve bu yapıların bazı elektronik özelliklerini incelemişlerdir [8]. Srivastava ve Misra $\mathrm{Li}_{\mathrm{n}} \mathrm{F}(\mathrm{n}=2-5)$ topaklarının teorik olarak lineer olmayan optik özelliklerini araştırmışlardır [4].

$\mathrm{Bu}$ çalışmada, lineer $\mathrm{Li}_{\mathrm{n}} \mathrm{F}(\mathrm{n}=1-8)$ topakları ile lineer olmayan $\mathrm{Li}_{\mathrm{n}} \mathrm{F}(\mathrm{n}=2-8)$ topakların kararlı yapılarının literatürde bilinmeyen bazı yeni fiziksel ve kimyasal özelliklerini ortaya çıkarmak için optik özellikleri üzerine sistematiksel bir çalışma yapılmıştır.

\section{KURAMSAL HESAPLAMLAR}

$\mathrm{Bu}$ çalışmada yapılan tüm hesaplamalar Gaussian 09W programı yardımıyla gerçekleştirilmiştir [17]. Lineer $\mathrm{Li}_{\mathrm{n}} \mathrm{F}(\mathrm{n}=1-8)$ topakların kararlı izomerik geometrik yapılarını elde etmek için B3LYP (Becke 3 LeeYang-Parr) $[18,19]$ teori seviyesinde flor atomu için efektif çekirdek potansiyeli içeren Los Alamos National Laboratory - 2 double zeta (Lanl2dz) baz setinde optimizasyon edilmişlerdir. İyonik yarıçapı büyük olan atomlarda çekirdeğe yakın bulunan elektronlara, yaklaşık olarak efektif çekirdek potansiyeli gibi davranılır. Bu davranış, iyonik yarıçapı büyük olan atomlar için önemli olan bazı rölatif etkileri içerir. Lanl2dz baz seti bunların en iyi bilineni olmasıyla beraber bir halojen atom katkılanmış lityum topaklarında deneysel verilere daha yakın sonuç verdiğinden kuramsal hesaplamalarımızda bu temel seti kullandık [3, 8, 20]. Optimizasyon hesaplamalarında farklı spin durumları göz önünde bulundurulmuştur. Lineer olmayan $\mathrm{Li} \mathrm{i}_{\mathrm{n}} \mathrm{F}(\mathrm{n}=2-8)$ topaklarında ise Ünal ve Kotan yaptığı çalışmada elde edilen global minimum geometrik yapı parametreleri kullanılarak DFT / B3LYP / Lanl2dz teori seviyesinden optimizasyon kararlılıkları tekrar teste tabi tutulmuştur. Lineer ve lineer olmayan flor-lityum topakların geometri optimizasyon sürecinde ilk adım olarak Berny algoritmasında yakınsama seviyeleri için öz uyumlu alan (SCF) elektronik yap1 hesaplamaları toplam enerjide $10^{-6}$ a.u., maksimum kuvvet $4.5 \times 10^{-5}$ a.u., maksimum yer değiştirme için $1.8 \times 10^{-3}$ a.u. ve RMS yer değiştirmesi için $1.2 \times 10^{-3}$ a.u. kriterleri seçilerek gerçekleştirilmiştir. İkinci adımda ise her bir topağın kendine has potansiyel enerji yüzeylerinde taban durumunu olup olmadığını doğrulamak için kızılötesi titreşim frekansları aynı metot ve baz setinde incelendi. Titreşim frekansı analizinde negatif frekans veya frekansların bulunmaması, elde edilen optimize yapının ilgili topağın potansiyel enerji yüzeyindeki minimuma karşılık geldiğini göstermiştir. Lineer optik (LO) ve lineer olmayan optik (NLO) parametreleri olan dipol momenti $(\mu)$, statik ortalama polarizebilitesi $(<\alpha>)$, anizotropik polarizebilite $(\Delta \alpha)$ ve birinci dereceden statik toplam moleküler hiperpolarizebilitesi $\left(\beta_{0}\right)$ gibi nicelikler sonlu-alan yaklaşımında [21] kullanılan ifadeler aşağıda verilmiştir:

$$
\begin{aligned}
& \mu=\left(\mu_{x}^{2}+\mu_{y}^{2}+\mu_{z}^{2}\right)^{1 / 2} \\
& \langle\alpha\rangle=\frac{1}{3}\left(\alpha_{x x}+\alpha_{y y}+\alpha_{z z}\right)
\end{aligned}
$$




$$
\begin{aligned}
& \Delta \alpha=\frac{1}{\sqrt{2}}\left[\left(\alpha_{x x}-\alpha_{y y}\right)^{2}+\left(\alpha_{y y}-\alpha_{z z}\right)^{2}+\left(\alpha_{z z}-\alpha_{x x}\right)^{2}\right]^{1 / 2} \\
& \beta_{0}=\left(\beta_{x}^{2}+\beta_{y}^{2}+\beta_{z}^{2}\right)^{1 / 2} ; \beta_{i}=\frac{3}{5}\left(\beta_{i i i}+\beta_{i i j}+\beta_{i k k}\right) ;(i \neq j \neq k=x, y, z)
\end{aligned}
$$

$i, j$ ve $k(=x, y$ ve $z)$ alt indisleri $\mu,\left\langle\alpha>, \Delta \alpha\right.$ ve $\beta_{0}$ bileşenlerinin kartezyen koordinat doğrultularını belirtmektedir ve 0.01 a.u.' luk bir elektrik alan şiddeti hassaslığında hesaplanmıştır.

\section{BULGULAR VE TARTIŞMA}

Lineer $\mathrm{Li}_{\mathrm{n}} \mathrm{F}(\mathrm{n}=1-8)$ topakların optimize olan kararlı izomerik yapıları Şekil 1' de gösterilmiştir. Şekil 1' de betimlenen bütün izomerik yapılar bundan sonra $n m$ olarak sınıflandırılacaktır. Bu sınıflandırmada, $n$ indisi lityum atomlarının sayısını, $m$ ise $a, b, c, d$,e harflerini içeren bir indis olup $a$ en düşük enerjili yapıyı temsil ederken ve $b$-e ise her bir topağa ait izomerlerinin kararlılık sırasını belirtmektedir. Şekil 2' de ise sadece lineer olmayan $\mathrm{Li}_{\mathrm{n}} \mathrm{F}(\mathrm{n}=2-8)$ topakların optimize olan en düşük enerjili geometrik yapıları sunulmuştur.

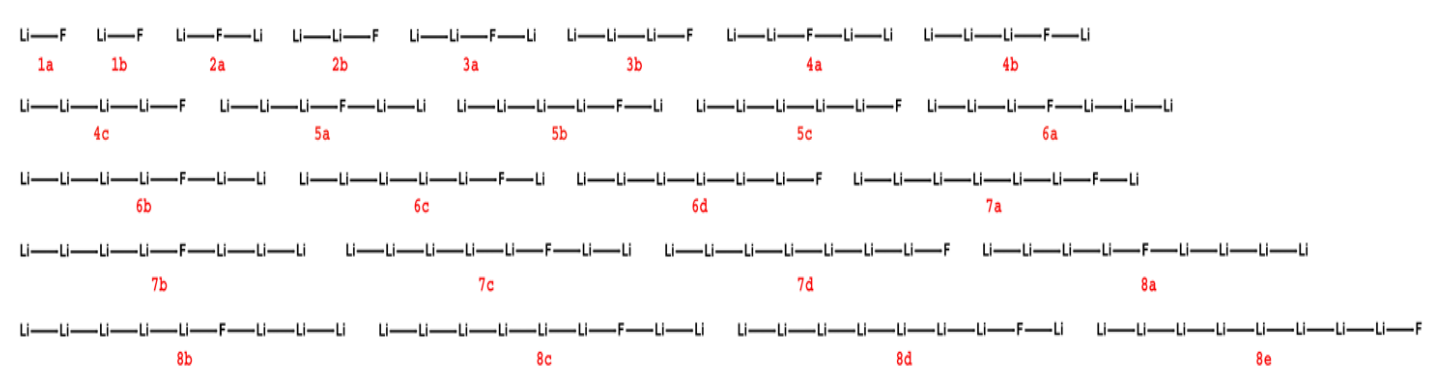

Şekil 1. Lineer $\mathrm{Li}_{\mathrm{n}} \mathrm{F}(\mathrm{n}=1-8)$ topaklarının kararlı izomerlerinin geometrik yapıları.

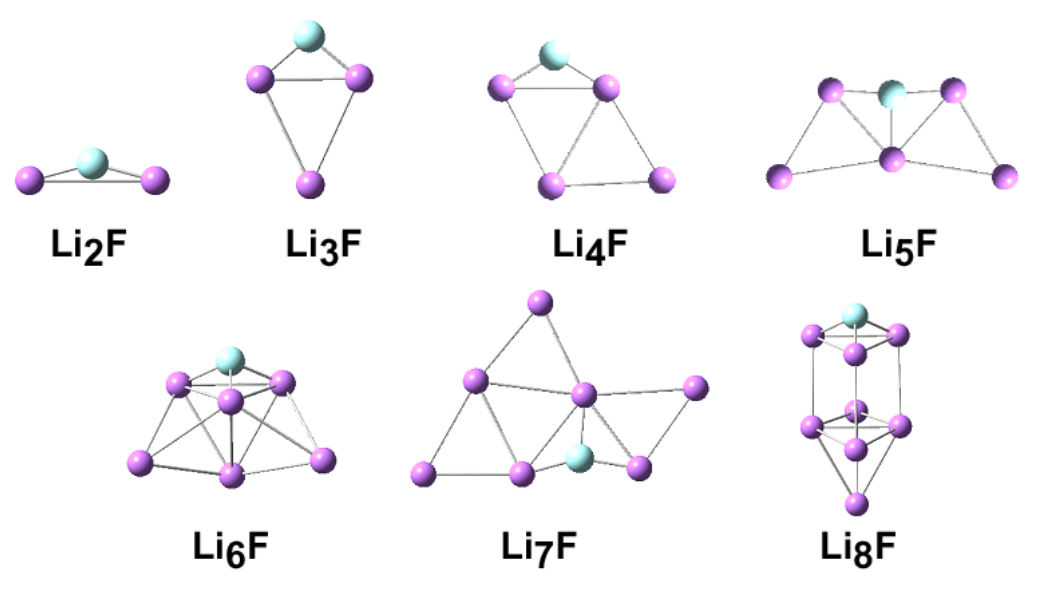

Şekil 2. Lineer olmayan $\mathrm{Li}_{\mathrm{n}} \mathrm{F}(\mathrm{n}=1-8)$ topaklarının en kararlı geometrik yapıları [8].

Lineer $\mathrm{Li}_{\mathrm{n}} \mathrm{F}(\mathrm{n}=1-8)$ topaklarının her bir topağına ait kararlı izomerlerinin simetri, çok katlılık, öz uyumlu alan enerji, sıfir nokta enerjisini içeren total enerji, Gibbs serbest enerji ve bu enerjilerin bağıl enerjileri ile HOMO-LUMO gap değerleri Tablo 1'de sunulmuştur. Tablo 1' den de görülmektedir ki, her bir topak en az iki izomer içermekte olup, lityum atom sayısı artmasına rağmen flor atomu en kararlı lineer flor-lityum topaklarında orta (merkez) konumu tercih etmiştir (Li-F hariç). Bununla beraber kararlı flor-lityum topaklarında lityum atom sayısında bağlı ortaklanmamış elektron sayısında artma eğilimi olduğu gözlenmiştir. $\mathrm{Li}_{4} \mathrm{~F}$ topağına kadar ortaklanmamış elektron sayısı kapalı çekirdek topaklarda en fazla bir iken, açık çekirdek topaklarda en fazla üç adet olduğu gözlenmiştir. Lityum atom sayısı dörtten fazla olan topaklarda ortaklanmamış elektron sayısı yedi adete kadar çıkmıştır. Lityum atom sayısının artmasıyla oluşan kararlı lineer flor-lityum topaklarda ortaklanmamış elektron sayısının artmasından dolayı yeni kararlı yapılar oluşmasına neden olmuştur. Fakat incelenen lineer flor-lityum topakların en düşük enerjili kararlı yapılar ( a yapıları, n=1-8) her zaman en az 
ortaklanmamış elektron sayısına sahip olan yapılar olduğu gözlenmiştir. Kapalı çekirdek topaklarda en kararlı yapılar $\mathrm{C}_{\infty \mathrm{v}}$ simetri grubunda olduğu gözlenirken, açık çekirdek topaklarda ise $\mathrm{D}_{\infty \mathrm{h}}$ simetrisini tercih ettikleri gözlenmiştir. Her bir topağa ait izomerlerinin kararlılık sıralaması öz uyumlu alan enerji, sıfır nokta enerjisini içeren total enerji ve Gibbs serbest enerji değerlerine göre sınıflandırılmıştır.

Lineer olmayan $\mathrm{Li}_{\mathrm{n}} \mathrm{F}(\mathrm{n}=2-8)$ topaklarının en düşük enerjili yapılarının simetri, çok katlılık, öz uyumlu alan enerji, sıfır nokta enerjisini içeren total enerji, Gibbs serbest enerji ve HOMO-LUMO gap değerleri Tablo 2' de verilmiştir. $\mathrm{Bu}$ yapılarda $\mathrm{Li}_{5} \mathrm{~F}$ ve $\mathrm{Li}_{7} \mathrm{~F}$ topakları hariç diğer tüm topaklarda flor atomu hep tepe konumunu tercih etmiştir. Flor atomunun iyonik yarıçapının lityum atomunun iyonik yarıçapından çok büyük olması sebebiyle katkılanan flor atomu saf lityum topakları ile çiftli tek-bağ yapma eğilimini tercih etmiş̧tir. Flor atomunun efektif çekirdek potansiyele sahip bir atom olması saf lityum topaklarının kararlılıklarını artırmasını da sağlamıştır. $\mathrm{Li}_{5} \mathrm{~F}$ ve $\mathrm{Li}_{7} \mathrm{~F}$ topakların da ise katkılanan flor atomu lityum atomları ile üçlü tek-bağ yapma eğilimi tercihinden ötürü kararlı saf lityum topaklarının geometrik yapısını bozarak daha kararlı bir fiziksel ve kimyasal topak olmalarını sağlamıştır. Lineer olmayan $\mathrm{Li}_{n} \mathrm{~F}(\mathrm{n}=2-8)$ topaklarının en kararlı yapılarına ait HOMO-LUMO gap değerleri göstermiştir ki, $\mathrm{Li}_{\mathrm{n}} \mathrm{F}(\mathrm{n}=3,5,7)$ topakları $\mathrm{Li}_{\mathrm{n}} \mathrm{F}(\mathrm{n}=2,4,6,8)$ komşu topaklarından daha kararlıklıdır. Bu kararlığının sebebi kapalı çekirdek topakları komşu açık çekirdek topaklarından daha yüksek bir gap enerjisine sahip olmasından dolayı ön görülmüştür $[2,3,8]$.

Lineer $\mathrm{Li}_{\mathrm{n}} \mathrm{F}(\mathrm{n}=1-8)$ topakları ile lineer olmayan $\mathrm{Li}_{\mathrm{n}} \mathrm{F}(\mathrm{n}=2-8)$ topaklarının en düşük enerjili kararlı yapıları kıyaslandığında, aynı moleküler formüle sahip lineer olmayan topaklar lineer topaklardan daha düşük rölatif enerjiye sahip oldukları görülmüştür. Bunun sebebi $\mathrm{Li}_{\mathrm{n}} \mathrm{F}$ topaklarının en düşük enerjili yapıları (LiF hariç) iki-boyutlu veya üç-boyutlu geometrik yapıları tercih etmesi eğiliminde olduğu düşünülmüştür.

Tablo 1. Lineer $\mathrm{Li}_{\mathrm{n}} \mathrm{F}(\mathrm{n}=1-8)$ topaklarının izomer, simetri, çok katlılık (Multi), moleküler enerji (E $\mathrm{S}_{\mathrm{SCF}}$ ), sıfır nokta enerjisini içeren total enerji $\left(\mathrm{E}_{\mathrm{T}}\right)$, Gibbs serbest enerji $(\Delta \mathrm{G})$, bağıl moleküler enerji $\left(\Delta \mathrm{E}_{\mathrm{SCF}}\right)$, bağıl total enerji $\left(\Delta \mathrm{E}_{\mathrm{T}}\right)$, bağıl Gibbs serbest enerji $(\delta \Delta \mathrm{G})$ ve HOMOLUMO gap (GapHL) verileri.

\begin{tabular}{|c|c|c|c|c|c|c|c|c|c|c|}
\hline Topak & İzomer & Simetri & Multi & $\mathbf{E}_{\text {SCF }}$ (a.u.) & $\mathbf{E}_{\mathbf{T}}$ (a.u.) & $\Delta G$ (a.u.) & $\Delta \mathrm{E}_{\mathrm{SCF}}$ (a.u.) & $\Delta \mathbf{E}_{\mathrm{T}}$ (a.u.) & $\delta \Delta \mathbf{G}$ (a.u.) & GapHL (a.u.) \\
\hline \multirow{2}{*}{$\mathrm{LiF}$} & $1 \mathrm{a}$ & $\mathrm{C}_{\infty \mathrm{V}}$ & 1 & -107.442 & -107.440 & -107.459 & 0.000 & 0.000 & 0.000 & \multirow[t]{2}{*}{0.204} \\
\hline & $1 b$ & $\mathrm{C}_{\infty \mathrm{v}}$ & 3 & -107.241 & -107.241 & -107.265 & 0.201 & 0.199 & 0.194 & \\
\hline \multirow{2}{*}{$\mathrm{Li}_{2} \mathrm{~F}$} & $2 \mathrm{a}$ & $\mathrm{D}_{\infty \mathrm{h}}$ & 2 & -114.997 & -114.995 & -115.018 & 0.000 & 0.000 & 0.000 & \multirow[t]{2}{*}{0.029} \\
\hline & $2 b$ & $\mathrm{C}_{\infty \mathrm{v}}$ & 4 & -114.720 & -114.719 & -114.745 & 0.277 & 0.276 & 0.273 & \\
\hline \multirow{2}{*}{$\mathrm{Li}_{3} \mathrm{~F}$} & $3 a$ & $\mathrm{C}_{\infty \mathrm{V}}$ & 1 & -122.517 & -122.512 & -122.540 & 0.000 & 0.000 & 0.000 & \multirow[t]{2}{*}{0.025} \\
\hline & $3 b$ & $\mathrm{C}_{\infty \mathrm{v}}$ & 3 & -122.439 & -122.436 & -122.468 & 0.078 & 0.076 & 0.072 & \\
\hline \multirow{3}{*}{$\mathrm{Li}_{4} \mathrm{~F}$} & $4 a$ & $\mathrm{D}_{\infty \mathrm{h}}$ & 2 & -130.034 & -130.028 & -130.060 & 0.000 & 0.000 & 0.000 & \multirow[t]{3}{*}{0.015} \\
\hline & $4 b$ & $\mathrm{C}_{\infty \mathrm{v}}$ & 2 & -130.026 & -130.021 & -130.055 & 0.008 & 0.007 & 0.005 & \\
\hline & $4 \mathrm{c}$ & $\mathrm{C}_{\infty \mathrm{v}}$ & 4 & -129.937 & -129.933 & -129.970 & 0.097 & 0.095 & 0.090 & \\
\hline \multirow{3}{*}{$\mathrm{Li}_{5} \mathrm{~F}$} & $5 a$ & $\mathrm{C}_{\infty \mathrm{v}}$ & 3 & -137.538 & -137.531 & -137.569 & 0.000 & 0.000 & 0.000 & \multirow[t]{3}{*}{0.011} \\
\hline & $5 b$ & $\mathrm{C}_{\infty \mathrm{V}}$ & 5 & -137.499 & -137.493 & -137.531 & 0.038 & 0.038 & 0.038 & \\
\hline & $5 c$ & $\mathrm{C}_{\infty \mathrm{v}}$ & 5 & -137.435 & -137.431 & -137.472 & 0.103 & 0.100 & 0.097 & \\
\hline \multirow{4}{*}{$\mathrm{Li}_{6} \mathrm{~F}$} & $6 a$ & $\mathrm{D}_{\propto \mathrm{h}}$ & 4 & -145.042 & -145.035 & -145.077 & 0.000 & 0.000 & 0.000 & \multirow[t]{4}{*}{0.008} \\
\hline & $6 b$ & $\mathrm{C}_{\infty \mathrm{v}}$ & 4 & -145.039 & -145.031 & -145.073 & 0.003 & 0.004 & 0.004 & \\
\hline & $6 c$ & $\mathrm{C}_{\infty \mathrm{v}}$ & 4 & -145.029 & -145.023 & -145.066 & 0.013 & 0.012 & 0.011 & \\
\hline & $6 d$ & $\mathrm{C}_{\infty \mathrm{v}}$ & 6 & -144.935 & -144.930 & -144.976 & 0.107 & 0.105 & 0.101 & \\
\hline \multirow{4}{*}{$\mathrm{Li}_{7} \mathrm{~F}$} & $7 \mathrm{a}$ & $\mathrm{C}_{\infty \mathrm{v}}$ & 3 & -152.551 & -152.554 & -152.592 & 0.000 & 0.000 & 0.000 & \multirow[t]{4}{*}{0.009} \\
\hline & $7 b$ & $\mathrm{C}_{\infty \mathrm{v}}$ & 5 & -152.543 & -152.535 & -152.582 & 0.008 & 0.009 & 0.010 & \\
\hline & $7 \mathrm{c}$ & $\mathrm{C}_{\infty \mathrm{v}}$ & 5 & -152.539 & -152.531 & -152.578 & 0.012 & 0.013 & 0.014 & \\
\hline & $7 d$ & $\mathrm{C}_{\infty \mathrm{v}}$ & 7 & -152.435 & -152.429 & -152.480 & 0.116 & 0.115 & 0.112 & \\
\hline \multirow{5}{*}{$\mathrm{Li}_{8} \mathrm{~F}$} & $8 \mathrm{a}$ & $\mathrm{D}_{\infty \mathrm{h}}$ & 6 & -160.044 & -160.036 & -160.087 & 0.000 & 0.000 & 0.000 & \multirow[t]{5}{*}{0.006} \\
\hline & $8 b$ & $\mathrm{C}_{\infty \mathrm{v}}$ & 6 & -160.043 & -160.035 & -160.085 & 0.001 & 0.001 & 0.002 & \\
\hline & $8 \mathrm{c}$ & $\mathrm{C}_{\infty \mathrm{v}}$ & 6 & -160.040 & -160.032 & -160.083 & 0.004 & 0.004 & 0.004 & \\
\hline & $8 d$ & $\mathrm{C}_{\infty \mathrm{v}}$ & 8 & -160.002 & -159.993 & -160.045 & 0.042 & 0.043 & 0.042 & \\
\hline & $8 \mathrm{e}$ & $\mathrm{C}_{\infty \mathrm{v}}$ & 8 & -159.936 & -159.929 & -159.984 & 0.108 & 0.107 & 0.103 & \\
\hline
\end{tabular}

Tablo 2. Lineer olmayan $\mathrm{Li}_{\mathrm{n}} \mathrm{F}(\mathrm{n}=1-8)$ en kararlı topaklarının simetri, çok katlılık (multi), moleküler enerji (E $\left.\mathrm{E}_{\mathrm{SCF}}\right)$, sıfır nokta enerjisi içeren total enerji $\left(\mathrm{E}_{\mathrm{T}}\right)$, Gibbs serbest enerji $(\Delta \mathrm{G})$ ve HOMO-LUMO gap (GapHL) verileri.

\begin{tabular}{ccccccc}
\hline Topak & Simetri & Multi & $\mathbf{E}_{\mathbf{S C F}}$ (a.u.) & $\mathbf{E}_{\mathbf{T}}$ (a.u.) & $\Delta \mathbf{G}$ (a.u.) & GapHL (a.u.) \\
\hline $\mathrm{Li}_{2} \mathrm{~F}$ & $\mathrm{C}_{2 \mathrm{v}}$ & 2 & -114.999 & -114.997 & -115.021 & 0.033 \\
$\mathrm{Li}_{3} \mathrm{~F}$ & $\mathrm{C}_{2 \mathrm{v}}$ & 1 & -122.532 & -122.527 & -122.553 & 0.083 \\
$\mathrm{Li}_{4} \mathrm{~F}$ & $\mathrm{C}_{\mathrm{s}}$ & 2 & -130.050 & -130.044 & -130.075 & 0.061 \\
\hline
\end{tabular}




\begin{tabular}{lllllll}
\hline $\mathrm{Li}_{5} \mathrm{~F}$ & $\mathrm{C}_{2 \mathrm{v}}$ & 1 & -137.595 & -137.587 & -137.618 & 0.075 \\
$\mathrm{Li}_{6} \mathrm{~F}$ & $\mathrm{C}_{\mathrm{s}}$ & 2 & -145.113 & -145.104 & -145.138 & 0.047 \\
$\mathrm{Li}_{7} \mathrm{~F}$ & $\mathrm{C}_{1}$ & 1 & -152.641 & -152.630 & -152.670 & 0.067 \\
$\mathrm{Li}_{8} \mathrm{~F}$ & $\mathrm{C}_{\mathrm{s}}$ & 2 & -160.176 & -160.164 & -160.202 & 0.046 \\
\hline
\end{tabular}

Lineer $\mathrm{Li}_{\mathrm{n}} \mathrm{F}(\mathrm{n}=1-8)$ topaklarının global minimum enerjili yapılarının dipol moment $(\mu)$, statik ortalama polarizebilite $(<\alpha>)$, anizotropik polarizebilite $(\Delta \alpha)$ ve birinci dereceden statik toplam moleküler hiperpolarizebilite $\left(\beta_{0}\right)$ parametreleri bu topakların lineer optik (LO) davranışlarını araştırmak için hesaplanmıştır. Lineer topaklar için hesaplanan LO aktiviteleri Tablo 3' de listelenmiştir.

Tablo 3. Lineer $\mathrm{Li}_{\mathrm{n}} \mathrm{F}(\mathrm{n}=1-8)$ topaklarının en kararlı yapıları için hesaplanan dipol moment $(\mu)$, statik ortalama polarizebilite $(<\alpha>)$, anizotropik polarizebilite $(\Delta \alpha)$ ve birinci dereceden statik toplam moleküler hiperpolarizebilite $\left(\beta_{0}\right)$ verileri.

\begin{tabular}{cccccr}
\hline Topak & İzomer & $\boldsymbol{\mu}$ (a.u.) & $<\boldsymbol{\alpha}>$ (a.u.) & $\Delta \boldsymbol{\alpha}$ (a.u.) & $\boldsymbol{\beta}_{\mathbf{0}}$ (a.u.) \\
\hline $\mathrm{LiF}$ & $1 \mathrm{a}$ & 2.48 & 7.52 & 3.08 & 217.01 \\
$\mathrm{Li}{ }_{2} \mathrm{~F}$ & $2 \mathrm{a}$ & 0.00 & 378.48 & 584.66 & 0.00 \\
$\mathrm{Li}_{3} \mathrm{~F}$ & $3 \mathrm{a}$ & 2.46 & 436.81 & 680.06 & 7178.93 \\
$\mathrm{Li}_{4} \mathrm{~F}$ & $4 \mathrm{a}$ & 0.00 & 759.93 & 1504.87 & 0.00 \\
$\mathrm{Li}_{5} \mathrm{~F}$ & $5 \mathrm{a}$ & 0.79 & 1112.27 & 2423.92 & 55309.30 \\
$\mathrm{Li}_{6} \mathrm{~F}$ & $6 \mathrm{a}$ & 0.00 & 1721.85 & 4114.80 & 0.00 \\
$\mathrm{Li}_{7} \mathrm{~F}$ & $7 \mathrm{a}$ & 1.88 & 2782.86 & 7066.74 & 787057.00 \\
$\mathrm{Li}_{8} \mathrm{~F}$ & $8 \mathrm{a}$ & 0.00 & 3133.17 & 8055.60 & 0.00 \\
\hline
\end{tabular}

Tablo 3'de sunulan dipol moment $(\mu)$ verileri göstermektedir ki, lityum atom sayısı çift olan lineer $\mathrm{Li}_{\mathrm{n}} \mathrm{F}$ $(\mathrm{n}=2,4,6,8)$ topakların dipol momentleri 0 a.u. iken lityum atom sayısı tek olan lineer $\mathrm{Li}_{\mathrm{n}} \mathrm{F}$ topakların $\mu$ değerleri 2.46 a.u. $\left(\mathrm{Li}_{3} \mathrm{~F}\right), 0.79$ a.u. $\left(\mathrm{Li}_{5} \mathrm{~F}\right)$ ve 1.88 a.u. $\left(\mathrm{Li}_{7} \mathrm{~F}\right)$ olarak hesaplanmıştır. Bu hesaplanan $\mu$ değerleri $\mathrm{LiF}$ molekülünün dipol momenti değerinden (2.48 a.u.) düşük olduğu gözlenmiştir. Bununla birlikte statik ortalama polarizebilite $(<\alpha>)$ ve anizotropik polarizebilite $(\Delta \alpha)$ değerleri lineer topaklarda lityum atom sayısı artmasıyla topağın hacmiyle orantılı artma eğiliminde olduğu görülmüştür. Lineer $\mathrm{Li}_{n} \mathrm{~F}(\mathrm{n} \geq 2)$ topakların hesaplanan $\langle\alpha>$ ve $\Delta \alpha$ verileri LiF moleküle ait 7.52 a.u $(<\alpha>)$ ve 3.08 a.u. $(\Delta \alpha)$ değerlerinden çok yüksek olduğu gözlenmiştir. Sert yumuşak asitler bazlar prensibine göre [22], düşük HOMO-LUMO gap (gapHL) enerjisine sahip sistemler daha sert ve polarizebiliteleri yüksek olabilirler. Lineer $\mathrm{Li}_{n} \mathrm{~F}(\mathrm{n} \geq 2)$ topakları $\mathrm{LiF}$ molekülünden $(0.204$ a.u.) çok daha düşük bir gapHL sahip olmaları, yüksek polarizebilite olmalarıla uyum içindedir. Bu uyum $\mu$ tanımlı olan lityum atom sayısı tek olan lineer topaklarının flor katkılanmış lityum topakların birinci dereceden statik toplam moleküler hiperpolarizebilite $\left(\beta_{0}\right)$ parametre değerlerinde de gözlenmiştir. $\operatorname{Li}_{n} \mathrm{~F}(\mathrm{n}=3,5,7)$ topaklarının $\beta_{0}$ değerleri, güçlü lineer optik (LO) davranışlarını ortaya koyacak kadar büyüktür (Tablo 3). Lineer $\mathrm{Li}_{7} \mathrm{~F}_{\text {ve }} \mathrm{Li}_{3} \mathrm{~F}$ topakların diğer lineer topaklara göre çok önemli LO davranışlar gösterdikleri gözlenmiştir.

Lineer olmayan optik (NLO) aktivitesi gösteren süperalkali topaklar alıcı-verici grup bağlı iki kutuplu moleküller iletişim ve optik veri depolama teknolojisi alanlarında son on yılda en çok araştırılan NLO malzemelerdir. Yariiletken tabakalı yapıda olan bu malzemeler organik ve/veya inorganik atomlar içeren topak sistemlerin NLO davranışları incelenmiştir. Bu tür sistemlerin $\pi$-konjugasyonun artması, NLO aktivitesinde artışa yol açmaktadır. Bir süperalkali topak sisteminde NLO aktivitesini yükseltmenin bir diğer yolu ise topağa alkali veya halojen atom (alıc1-verici grup) eklemektir. NLO özelliklerini öngörebilmek için hesaplanan $\mu,\langle\alpha>$, $\Delta \alpha$ ve $\beta_{0}$ parametrelerini merkezi atom ve merkezi atoma bağlı atomlar artırabilir ya da azaltabilir. Ancak bu parametreler tüm negatif ve pozitif polarizebilite katkılarının toplamından elde edilerek NLO özellikleri öngörülebilir. Lineer olmayan $\mathrm{Li}_{n} \mathrm{~F}(\mathrm{n}=2-8)$ topaklarının en düşük enerjili yapılarının NLO davranışlarını öngörebilmek için $\mu,\langle\alpha\rangle, \Delta \alpha$ ve $\beta_{0}$ parametre değerleri hesaplanmıştır. Lineer olmayan $\operatorname{Li}_{n} \mathrm{~F}(\mathrm{n}=2-8)$ topakları için hesaplanan dipol moment ve polarizebilite parametre değerleri Tablo 4 ' de sunulmuştur.

Tablo 4. Lineer olmayan $\mathrm{Li}_{\mathrm{n}} \mathrm{F}(\mathrm{n}=2-8)$ topaklarının en kararlı yapıları için hesaplanan dipol momenti $(\mu)$, statik ortalama polarizebilite $(<\alpha>)$, anizotropik polarizebilite $(\Delta \alpha)$ ve birinci dereceden statik toplam moleküler hiperpolarizebilite $\left(\beta_{0}\right)$ verileri.

\begin{tabular}{cccrr}
\hline Topak & $\boldsymbol{\mu}$ (a.u.) & $<\boldsymbol{\alpha}>$ (a.u.) & $\Delta \boldsymbol{\alpha}$ (a.u.) & $\boldsymbol{\beta}_{\mathbf{0}}$ (a.u.) \\
\hline $\mathrm{Li}_{2} \mathrm{~F}$ & 0.16 & 347.04 & 527.63 & 10118.00 \\
$\mathrm{Li}_{3} \mathrm{~F}$ & 0.45 & 183.44 & 59.68 & 747.75 \\
$\mathrm{Li}_{4} \mathrm{~F}$ & 0.64 & 290.08 & 187.80 & 6195.84 \\
$\mathrm{Li}_{5} \mathrm{~F}$ & 0.43 & 351.93 & 171.37 & 960.43 \\
$\mathrm{Li}_{6} \mathrm{~F}$ & 0.26 & 446.81 & 219.24 & 6196.54 \\
$\mathrm{Li}_{7} \mathrm{~F}$ & 0.60 & 548.03 & 347.20 & 7994.32 \\
$\mathrm{Li}_{8} \mathrm{~F}$ & 0.13 & 539.21 & 109.46 & 1702.05 \\
\hline
\end{tabular}


Lineer olmayan $\mathrm{Li}_{n} \mathrm{~F}(\mathrm{n}=2-8)$ topakların dipol moment $(\mu)$ değerleri 0.13 a.u. $\left(\mathrm{Li}_{8} \mathrm{~F}\right)$ ile 0.64 a.u. $\left(\mathrm{Li}_{4} \mathrm{~F}\right)$ arasında olduğu hesaplanmıştır. Bu topakların $\mu$ değerleri LiF molekülünün dipol momenti değerinin (2.48 a.u.) çok altında olduğu görülmüştür. Fakat $\mathrm{Li}_{n} \mathrm{~F}(\mathrm{n}=2-8)$ topakların statik ortalama polarizebilite $(<\alpha>)$ ve anizotropik polarizebilite $(\Delta \alpha)$ parametre değerleri $\operatorname{LiF}(<\alpha>=7.52$ a.u ve $\Delta \alpha=3.08$ a.u.) molekülünden çok büyüktür ve $\langle\alpha\rangle$ parametre değerleri yaklaşık olarak 183 a.u. ile 548 a.u. arasında değişkenlik gösterirken $\Delta \alpha$ parametre değerleri ise yaklaşık olarak 60 a.u. ile 528 a.u.değişkenlik gözlenmiştir. Lineer olamayan $\mathrm{Li}_{\mathrm{n}} \mathrm{F}(\mathrm{n}=2-8)$ topakların HOMO-LUMO gap (gapHL) değerleri 0.033 a.u. ile 0.083 a.u. aralığında hesaplanmıştır. LiF için hesaplanan gapHL 0.204 a.u.' dur. $\mathrm{Li}_{\mathrm{n}} \mathrm{F}(\mathrm{n}=2-8)$ topakların gapHL değerleri yaklaşık olarak $\mathrm{LiF}$ molekülünün üçte biri ile altıda biri oranında olmasından dolayı bu topakların kimyasal yumuşaklığı yüksek olacağından topak içi oluşan yük transferi kolaylıkla yönlendirilebilir ve bu sayede bu topaklar yüksek polarizebilitere sahip olabilirler. Tablo $4^{\prime}$ de verilen birinci dereceden statik toplam moleküler hiperpolarizebilite $\left(\beta_{0}\right)$ değerlerine göre sirasıyla lineer olamayan $\mathrm{Li}_{2} \mathrm{~F}, \mathrm{Li}_{7} \mathrm{~F}, \mathrm{Li}_{6} \mathrm{~F}$ ve $\mathrm{Li}_{4} \mathrm{~F}$ topakları çok dikkat çekici bir NLO aktivitesi göstermiştir.

\section{SONUÇLAR}

Lineer $\mathrm{Li}_{n} \mathrm{~F}(\mathrm{n}=1-8)$ topaklarının düşük enerjili kararlı yapıları ilk kez hibrit-Yoğunluk Fonksiyonel Teorisi (h-DFT) yöntemi kullanılarak elde edilmiştir. Lineer $\mathrm{Li}_{\mathrm{n}} \mathrm{F}(\mathrm{n}=1-8)$ topakları ile lineer olmayan $\mathrm{Li}_{\mathrm{n}} \mathrm{F}(\mathrm{n}=2$ 8) topaklarına ait global minimum kararlı yapılarının lineer optik (LO) ve lineer olmayan optik (NLO) özellikleri hakkında detaylı bilgiler elde etmek için dipol moment $(\mu)$, statik ortalama polarizebilite $(\langle\alpha\rangle)$, anizotropik polarizebilite $(\Delta \alpha)$ ve birinci dereceden statik toplam moleküler hiperpolarizebilite $\left(\beta_{0}\right)$ parametreleri aynı yöntemde tanımlanmaya çalışılmıştır. Sunulan kuramsal polarizebilite bilgileri ışığı altında özellikle lineer $\mathrm{Li}_{7} \mathrm{~F}$ topağ 1 ile $\mathrm{Li}_{3} \mathrm{~F}$ topakları yanı sıra lineer olamayan özelikle $\mathrm{Li}_{2} \mathrm{~F}$ topağ ile $\mathrm{Li}_{7} \mathrm{~F}, \mathrm{Li}_{6} \mathrm{~F}$ ve $\mathrm{Li}_{4} \mathrm{~F}$ topakları kayda değer yüksek optik davranışlar göstermişlerdir. Bu çalışma gelecekte yapılacak LO ve NLO materyallerinin geliştirilmesinde tek flor atomu katkılanmış lityum topakların potansiyel uygulamalarının artması amaçlanmıştır.

\section{KAYNAKLAR}

[1] Linden D. (1995). Handbook of Batteries, $2^{\text {nd }}$ ed., McGraw-Hill, New York.

[2] Şentürk, Ş. (2011). A Density Functional Study of $\mathrm{Li}_{n} \mathrm{Cl}$ (n=1-7) Clusters. Z. Naturforsch. A, 66, 372-376.

[3] Şentürk Ş., Ünal, A., \& Kalfa, O.M. (2013). Density functional study of bromine doped lithium clusters. Comput. Theor. Chem., 1023, 46-50.

[4] Srivastava, A.K., \& Misra, N. (2015). Nonlinear optical behavior of $\operatorname{Li}_{n} \mathrm{~F}(n=2-5)$ superalkali clusters. $J$. Mol. Model., 21, 305.

[5] Milovanović, M., Veličković, S., Veljković, F., \& Jerosimić, S. (2017). Structure and stability of small lithiumchloride $\mathrm{Li}_{n} \mathrm{Cl}_{m}{ }^{(0,1+)}(n \geq m, n=1-6, m=1-3)$ clusters. Phys. Chem. Chem. Phys., 19, 30481-30497.

[6] Srivastava, A.K., \& Misra, N. (2016). Remarkable NLO responses of hyperalkalized species: the size effect and atomic number dependence. New J. Chem., 40, 5467-5472.

[7] Velickovic, S.R., Koteski, V.J., Belosevic Cavor, J.N., Djordjevic, V.R., Cveticanin, J.M., Djustebek, J.B., Veljkovic, M.V., \& Neskovic, O.M. (2007). Experimental and theoretical investigation of new hypervalent molecules $\mathrm{Li}_{n} \mathrm{~F}$ (n=2-4). Chem. Phys. Lett., 448, 151-155.

[8] Ünal, A., \& Kotan, B. (2018). A DFT based study of geometries, stabilities and electronic properties of $\mathrm{Li}_{n} \mathrm{~F}(n=1-8)$ clusters. Main Group Chem., 17, 267-272.

[9] Dustebek, J., Velickovic, S.R., Veljkovic, F.M., \& Veljkovic, M.V. (2012). Production of heterogeneous superalkali clusters $\mathrm{Li}_{\mathrm{n}} \mathrm{F}(\mathrm{n}=2-6)$ by Knudsen cell Mass Spectrometry. Dig. J. Nanomater Bios., 7, $1365-$ 1372.

[10] Lanaro, G., \& Patey, G.N. (2017). Crystal structures of model lithium halides in bulk phase and in clusters. J. Chem. Phys., 146, 154501.

[11] Moreira, N.L., Brito, B.G.A., Rabelo, J.N.T., \& Cândido, L. (2016). Quantum Monte Carlo study of the energetics of small hydrogenated and fluoride lithium clusters. J. Comput. Chem., 37, 1534-1536. 
[12] Milonavić, M.Z., \& Jerosimić, S.V. (2014). Theoretical investigation of geometry and stability of small lithium-iodide $\mathrm{Li}_{n} \mathrm{I}$ (n=2-6) clusters. Int. J. Quantum Chem., 114, 192-208.

[13] Gutsev, G.L., \& Boldryev, A.I. (1981). DVM-X $\alpha$ calculations on the ionization potentials of $\mathrm{MX}_{k+1}{ }^{-}$ complex anions and the electron affinities of $\mathrm{MX}_{k+1}$ "superhalogens". Chem. Phys., 56, 277-283.

[14] Gutsev, G.L., \& Boldryev, A.I. (1982). DVM X $\alpha$ calculations on the electronic structure of "superalkali" cations, Chem. Phys. Lett., 92, 262-266.

[15] Rehm, E., Boldryev, A.I ., \& Schleyer, P.v.R.(1992). Ab initio study of superalkalis. First ionization potentials and thermodynamic stability. Inorg. Chem. 31, 4834-4842.

[16] Li, Y., \& Wu, D. (2010). Theoretical study on static first hyperpolarizabilities of hypervalent compounds $\mathrm{F}_{n} \mathrm{Li}_{n+1}(n=1-3)$. Gaodeng Хиехіао Ниахие Хиеbao, 31, 1811-1814.

[17] Frisch, M.J., et al., Gaussian 09 Revision A.1, Gaussian Inc., Wallingford, CT, Gaussian, Inc. 2009.

[18] Becke, A. D. (1993). Density-functional thermochemistry. III. The role of exact exchange. J. Chem. Phys., 98, 5648-5652.

[19] Lee, C., Yang, W., \& Parr, R.G. (1988). Development of the Colle-Salvetti correlation-energy formula into a functional of the electron density. Phys. Rev. B, 37, 785-789.

[20] Kotan, B. (2018). Flor katkılı lityum topaklarının en düşük enerjili yapılarının araştırılması. Yüksek Lisans Tezi, Bilecik Şeyh Edebali Üniversitesi, Fen Bilimleri Enstitüsü, Bilecik.

[21] Cohen, H.D., \& Roothaan, C.C. (1965). Electric Dipole Polarizability of Atoms by the Hartree-Fock Method. I. Theory for Closed-Shell Systems. J. Chem. Phys., 43, S34-S39.

[22] Pearson, R. G. (1963). Hard and Soft Acids and Bases. J. Am. Chem. Soc., 85, 3533-3539. 